S0081

\section{Changes in pornography use and sexual behaviour during lockdown}

\author{
M. Koós, B. Bőthe, O. Király, B. Paksi and Z. Demetrovics \\ Institute Of Psychology, ELTE Eötvös Loránd University, Budapest, \\ Hungary \\ ${ }^{\star}$ Corresponding Author. \\ doi: 10.1192/j.eurpsy.2021.94
}

COVID-19 pandemic and the related restrictions had a significant impact on the living and working conditions as well as the everyday behavior and mental health condition. Aim of the current analysis was to examine the impact of the input-deprived circumstances on the sexual life characteristics. An online survey carried out after a few weeks of the first nation-wide lockdown was enacted in Hungary. 1,755 persons participated in the first wave (50.4\% males). Relationship and sexual life satisfaction, sexual intercourse and masturbation frequency were assessed with additional single-item questions about the subjective change (5-pont Likert scale; $1=$ "decreased significantly"; $5=$ "increased significantly) in these characetristics since the epidemiological restrictions had been introduced. Furthermore, several potential protective and risk factors were measured (depressive symptoms, perceived stress, loneliness, general well being, intolerance of uncertainty, sensation seeking, and COVID-19 related health anxiety). Linear regression models were calculated to assess which of the former variables predict the subjective changes of one's sexual life. The analyses resulted in weak standardized coefficients. The subjective change in relationship satisfaction (mean 3.20, SD 0.94) and sexual satisfaction (mean 2.82 , SD 0.73 ) were predicted positively by general wellbeing $(\beta=.11-0.25, p<.01)$, and negatively by loneliness $(\beta=-.14$ $0.19, \mathrm{p}<0.01)$. Loneliness predicted negatively the subjective change in sexual intercourses (mean 2.75, SD 0.89) and masturbation frequency (mean 2.89, SD 0.84) $(\beta=-.10-.12, \mathrm{p}<.01)$, while sensation seeking had no effect $(\beta=-.09, \mathrm{p}<.01)$. The COVID-19 related health anxiety predicted negatively only the change in sexual life frequency $(\beta=-.07, p<.05)$. The explained variances were rather small $(1.7 \%-11.8 \%)$.

Disclosure: No significant relationships.

Keywords: Sexual Behavior; sexual life satisfaction; quarantine; sexual life frequency

\section{S0080 \\ Internet addiction and mental disorders: Clinical effects by self-distancing}

\section{G. Bersani}

Department Of Medico-surgical Sciences And Biotechnologies, Sapienza University of Rome, Latina, Italy

doi: 10.1192/j.eurpsy.2021.95

We face today the huge and very rapid worldwide growth of behavioural issues related to the use of Internet. The definition of Problematic Internet Use (PIU) refers to new behavioural patterns that can potentially affect in variable degree, from mild to extremely severe, both individual and social wellbeing. PIU is strongly increasing in people affected by different forms of mental disorders and personality disorders, often inducing substantial changes in their clinical phenomenology, with consequent emergence of new symptom and course profiles. On the other side, PIU represents itself with growing frequency as a factor with high potential of inducing progressive psychological and behavioural impairment, with possible negative outcome on personal and psychosocial wellbeing and adjustment, also potentially leading to the development of new specific forms of psychopathology. Among PIU patterns, Internet Addiction (IA) plays a central role, due to its wide diffusion and behavioural as well as interpersonal and social consequences. The worldwide COVID 19 epidemics induced limitations in direct social relationships, such as social distancing, appear to lead to changes of patterns of IA, for an increase of time spent in addictive behaviour and a further reduction of research of interpersonal contacts. Obsessive-compulsive and autistic-like behaviour are differently reinforced by the combined effect of compulsory self distancing and general health concern, but also possibly induced in previously not affected subjects. Anxiety and mood reactivity also contributes to maladjustment profiles. Further evidences and new guide-lines are requested to face this novel and multifactorial social and clinical phenomenon.

Disclosure: No significant relationships.

Keywords: Internet addiction; covid 19; psychopathology

\section{Implementing alternatives to coercion in mental health care}

\section{S0081}

\section{The WPA programme on implementing alternatives to coercion}

\section{H. Herrman}

Centre For Youth Mental Health, Orygen and University of Melbourne, Parkville, Australia

doi: 10.1192/j.eurpsy.2021.96

Abstract Body: The call for alternatives to coercion in mental health care is growing both within the profession and among people with lived experience of coercion in mental healthcare. There is widespread agreement that coercive practices are over-used. Considerable work is warranted across the mental health sector and in communities and governments to ensure that people living with mental disorders and psychosocial disabilities uniformly have access to high-quality care and support that meet their needs and respect their personhood and human rights. The question of whether coercive interventions can ever be justified as part of mental health treatment, to protect rights holders' own interests or on other grounds, is highly contested. WPA issued a Position Statement and Call to Action in 2020: Implementing Alternatives to Coercion: A Key Component of Improving Mental Health Care after extensive consultation and review. The purpose is (1) to recognize the substantive role of psychiatry in implementing alternatives to coercion in mental health care and (2) to support action in this regard, essential to improving mental health treatment and care in all countries. The Statement recognises the diversity of views and experiences among mental health professionals, people with lived experience and their families and carers. This initial step is the beginning of a longer-term process, which requires continued engagement with WPA member societies, people with lived experience, families and other partners to encourage and support the 
implementation of alternatives to coercion in mental health care. https://www.wpanet.org/alternatives-to-coercion

Disclosure: No significant relationships.

\section{S0081a}

Service user perspectives on coercion in mental health

A. Nomidou

Secretary General, GAMIAN-Europe, Brussels, Belgium

doi: 10.1192/j.eurpsy.2021.97

GAMIAN-Europe is a patient-driven pan-European organisation, representing and advocating for the interests and rights of persons with psychosocial disabilities.

While recovery is a deeply personal journey it is also a product of interaction facilitated or impeded through the dynamic interplay of many forces, such as among characteristics of the individual, of the environment and of the exchange. To move recovery forward, recovery-oriented systems in recovery-facilitating environments are needed. Mental health professionals can either facilitate or hinder this journey. Service users and families want to feel they are more than their medical concerns, more than 'the suicidal' in room five. Respecting individuals and their human rights, active and engaged listening, including patients in their own healing plan, promoting wellness and engaging with compassion build trust between patients and health care professionals, leading to willingness to follow through with care plans. At the same time, by creating emotional connections and environments, not only can frequent burnouts be prevented, but productivity can be increased.

Disclosure: No significant relationships.

\section{Nutritional psychiatry}

\section{S0085}

Food for mood: Relevance of nutritional Omega-3 fatty acids for depression and anxiety

\section{S. Laye}

Inrae, Univ Bordeaux, NutriNeuro, Bordeaux, France doi: 10.1192/j.eurpsy.2021.98

Polyunsaturated fatty acids (PUFAs) are essential fatty acids which are provided to the body through the diet. The brain is one of the richest organs in the body and has a high need in PUFAs. There are 2 main families of PUFAs, n-3 (or omega 3) and n-6 (or omega6). While it is quite easy to find n-6 PUFAs in westernized diets, the need in n-3 PUFAs is poorly reached, leading to decreased level of docosahexaenoic acid (DHA) in the brain. In humans, poor levels of blood n-3 PUFAs and brain DHA are associated to a higher prevalence of cognitive disorders and depression. However, the mechanisms underlying the effect of DHA on brain functions are poorly understood. Using mice models of n-3 PUFAs dietary deficiency or supplementation, we revealed that in the brain, DHA regulate neuroinflammatory pathways, in particular through its effect on microglia, the main innate immune system cell in the brain. In addition, n-3 PUFAs are key actors of ndocannabinoid- dependent synaptic plasticity. While neuroinflammation and eCBdependent synaptic plasticity are crucial to cognition and emotional behaviour alterations, our results bring to the clinical scene the importance of controlling dietary n-3 PUFAs to protect the brain from the adverse effect of stres or inflammation. Altogether, our work brings a better comprehension of how dietary n-3 PUFAs participate to brain physiology and protect from the development of mood and cognitive disorders. It opens new avenues for the use of these lipids in the protection and treatment of brain diseases.

Disclosure: No significant relationships.

Keywords: omega-3; synaptic plasticity; neuroinflammation; depression; anxiety; microglia

Novel pharmacotherapeutic strategies for regaining control over alcohol intake in alcohol use disorder

\section{S0086}

\section{Role of oxytocin in modulating addictive behaviour}

\section{P. Bach, A. Koopmann and F. Kiefer}

Department Of Addictive Behaviour \& Addiction Medicine, Central Insitute of Mental Health, Mannheim, Germany

${ }^{\star}$ Corresponding Author.

doi: 10.1192/j.eurpsy.2021.99

Background: The brain oxytocin system is involved in a wide range of addictive behaviors, inhibiting prime- and cue-induced relapse in preclinical models of substance use disorders. Animal studies linked oxytocin's effects on drug ingestion to modulation of neurotransmission in the nucleus accumbens (NAc). We set out to investigate whether oxytocin can modulate alcohol cue-induced functional connectivity between the brain reward system and cortical regions.

Methods: Fifteen male heavy social drinkers were enrolled in a randomized double-blind placebo-controlled cross-over functional magnetic resonance imaging study (fMRI) investigating the effect of 24 IU oxytocin on alcohol cue-modulated functional connectivity. Results: Results of the functional connectivity analyses show that oxytocin application significantly reduced connectivity between the NAc and cuneus and thalamo-occipital connectivity, while enhancing connectivity between the paracingulate gyrus and precentral gyrus (tow-sided seed-level false discovery rate $\mathrm{p}_{\mathrm{FDR}}<0.05$ ). These effects were specific to the alcohol presentation and were absent during processing of neutral pictures. In addition, the NAc-cuneus connectivity significantly correlated with subjective alcohol cueinduced craving during the scanning session $(\mathrm{r}=0.538, \mathrm{p}=0.024)$. Conclusion: Results provide initial evidence for condition-specific and significant attenuation of NAc connectivity by oxytocin in a sample of heavy social drinkers that was related to lower subjective alcohol craving during the fMRI task. Oxytocin-induced attenuation of NAc connectivity was specific to processing alcohol stimuli and might reflect an attenuation of alcohol-cue saliency by oxytocin that could lead to a reduction of the sensitivity towards the appetitive aspects of alcohol cues.

Disclosure: No significant relationships.

Keywords: alcohol use disorder; Funtional Magnetic Resonance Imaging; Psychopharmacology; oxytocin 